\title{
Enhancing the Resolution and Sensitivity of STEM by Aberration Correction
}

\author{
N. D. Browning ${ }^{1}$, K. Sun ${ }^{1}$, R. F. Klie ${ }^{1}$, J. Liu ${ }^{2}$, M. M. Disko ${ }^{3}$, P. D. Nellist ${ }^{4}$, N. Dellby ${ }^{4}$, O. L. \\ Krivanek $^{4}$ \\ ${ }^{1}$ University of Illinois, Department of Physics, 845 W. Taylor St, Chicago, IL 60607, USA. \\ ${ }^{2}$ Monsanto Company, 800 N. Lindbergh Avenue, U1E, St Louis, MO 63167, USA. \\ ${ }^{3}$ ExxonMobil Res. \& Eng. Corp, Corporate Strategic Research, Annandale, NJ 08801. USA \\ ${ }^{4}$ Nion Company, $11028^{\text {th }}$ Street, Kirkland, WA 98033, USA
}

The ability to quantify the atomic structure, composition and local bonding at interfaces and defects is key to developing a fundamental understanding of the properties of many nanoscale systems. The combination of Z-contrast imaging and electron energy loss spectroscopy (EELS) in the scanning transmission electron microscope (STEM) [1] provides the ability to generate precisely this type of characterization. For these techniques, the spatial resolution is limited primarily by the probe size and the sensitivity is limited by the probe current. Enhancing the performance of the microscope and our ability to characterize the key properties relevant to the development of nanoscale materials, devices and processes therefore requires only a modification of the electron optics for probe formation.

Improvements in the probe size and probe current have recently been afforded by the advent of aberration correction [2]. Cs-correctors can now be purchased to upgrade the performance of $100 \mathrm{kV}$ dedicated VG STEM, pushing the probe size close to $0.12 \mathrm{~nm}$. Provided the room and intrinsic microscope instabilities can be removed, this probe size translates to an image resolution at $100 \mathrm{kV}$ that is comparable with the best STEM resolution at $200 \mathrm{kV}$ [3]. This improvement in resolution is coupled with an increase in probe current, a smaller energy spread of the beam (achieved by using a cold field-emission source rather than a Schottky source), and a lower accelerating potential; all features that will improve the analysis of "real" nanoscale systems.

One application where the decrease in probe size and increase in probe current can significantly increase our understanding of nanoscale phenomena is in the area of supported heterogeneous catalysts [4,5]. Here, understanding the origin of the activity and selectivity of a given catalytic system requires an intimate understanding of this metal-support interaction as a function of cluster size and reduction/oxidation treatments. For example, figure 1 shows an image of a larger $(\sim 200 \mathrm{~nm})$ copper particle in a $\mathrm{Cu} / \mathrm{Al}_{2} \mathrm{O}_{3}$ catalyst calcined at $1073 \mathrm{~K}$. Image contrast and the energy loss spectra shown in figure $1 \mathrm{~b}$, indicate that a covering oxide layer forms on this particle despite being heated in flowing hydrogen, i.e. a highly reducing atmosphere. Although the oxide layer can be observed in these larger particles, the sensitivity of the current microscope (JEOL 2010F [3]) does not allow the fine-structure of the edge to be analyzed in the particles $\sim$ few nanometers in size to determine whether a crystalline oxide is formed or whether the signal originates simply from adsorbed oxygen on the surface.

An aberration corrected $100 \mathrm{kV}$ dedicated STEM will significantly improve the characterization of these systems. Although in its infancy, the VG HB601 $\mathrm{C}_{\mathrm{s}}$ corrected STEM at UIC will be used primarily for the study of these systems. Initial results from the factory tests of the corrector are shown in figure 2 . Here a resolution of $\sim 0.118 \mathrm{~nm}$ is achieved with an objective aperture of 22 mrad and a probe current $\sim 60 \mathrm{pA}$. Both the probe size and the current are improved over what is currently available in the JEOL $2010 \mathrm{~F}$ at UIC $(0.13 \mathrm{~nm}$ probe size and $\sim 40 \mathrm{pA}$ probe current $)$. In 
this presentation, the latest results from the corrected STEM will be discussed with particular attention being paid to the practical advantages/disadvantages of such a system over a conventional TEM/STEM instrument [6].

\section{References}

[1] N. D. Browning, M. F. Chisholm, and S. J. Pennycook, Nature 366 (1993) 143.

[2] N. Dellby et al, J. Electron Microscopy 50 (2001) 177.

[3] E. M. James and N. D. Browning, Ultramicroscopy 78 (1999) 125.

[4] R. F. Klie et al, J. Catalysis 205 (2002), 1

[5] K. Sun et al, in press Applied Catalysis B

[6] This work was sponsored in part by the National Science Foundation under grant numbers DMR 9601792 and DMR 007364, by Monsanto Company, and by the Petroleum Research Fund.
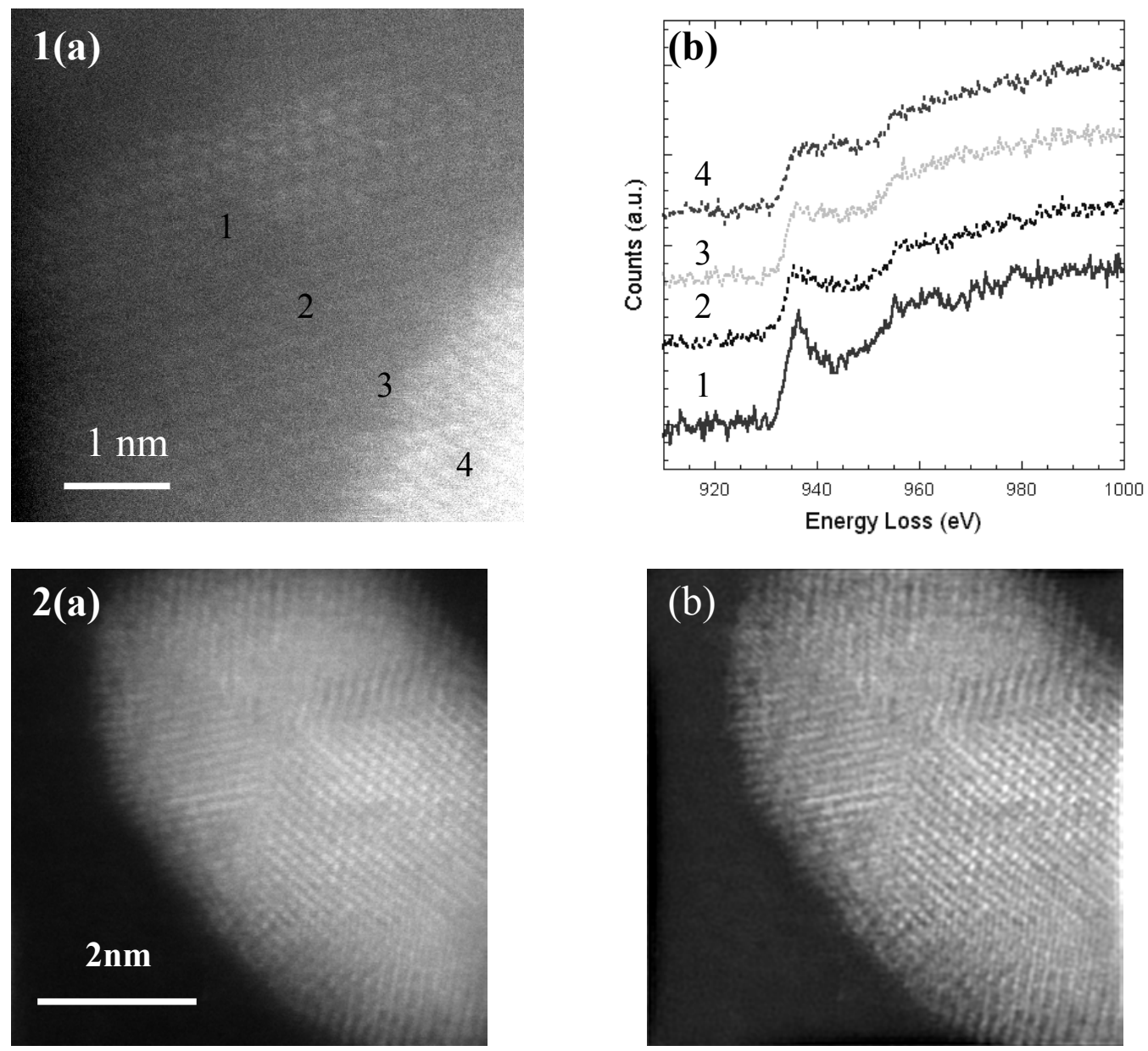

Figure 1: (a) Z-contrast image of a $200 \mathrm{~nm} \mathrm{Cu}$ particle on an $\mathrm{Al}_{2} \mathrm{O}_{3}$ support obtained on the JEOL 2010F. The surface shows a lower intensity, indicative of the formation of an oxide, which is confirmed by the presence of a white line in the copper L-edge spectrum (b).

Figure 2: Figure 2: Z-contrast image of an Au particle, (a) raw data, (b) low-pass Fourier filtered to improve fringe visibility. The largest observable spacing corresponds to the $0.23 \mathrm{~nm}$ (111) fringes. Fringes with much lower spacings, such as the $0.118 \mathrm{~nm}(222)$ fringes, are also visible. 